\title{
A prospective observational study to compare the efficacy and safety of spinal Vs general anesthesia for laparoscopic cholecystectomy
}

\author{
Subbiah V. ${ }^{1}$, Palaniappan S.K. ${ }^{2}$ \\ ${ }^{1}$ Dr. Venkatesh Subbiah, Assistant Professor. General Surgery, ${ }^{2}$ Dr. Selva Kumar Palaniappan, Assistant Professor, \\ Anaesthesia; both authors are affiliated with Velammal Medical College, Madurai, Tamil Nadu, India. \\ Corresponding Author: Dr. Selva kumar Palaniappan, Assistant Professor, Department of Anaesthesia, Velammal \\ Medical College, Madurai. Velammal Village, Anuppanadi, Near Chinthamani Toll Gate, Madurai, Tamil Nadu, India, \\ Email : selvakumarrevathy@gmail.com
}

\begin{abstract}
Background: Laparoscopic cholecystectomy is usually performed under General anesthesia, but in recent times many studies have proposed spinal anesthesia as a cost-effective alternative mode of anesthesia, specially for resource poor settings with comparable efficacy, safety. But there is a scarcity of literature on the subject. Hence the current study was conducted with an objective of comparing the efficacy and safety of spinal anesthesia, as compared to general anesthesia. Materials and Methods: The current study was prospective observational study, conducted in the department of general surgery and anesthesia, Velammal medical college, Madurai between April to December 2017. The study has included adult patients more than 18 years of age, with ASA grade I and II, posted for laparoscopic cholecystectomy. All the patients in the study period were administered with anesthesia as per their preference after explaining the pros and cons of each procedure. Results: A total of 73 subjects were included in the final analysis, with 28 subjects in spinal anesthesia group and 45 subjects in general anesthesia group. The median postoperative VAS score was statistically significantly lesser in spinal anesthesia group till 4-hour post-operative period, compared to general anesthesia group. After 4 hours the median VAS score was comparable between two groups. The ease of performing surgery score was similar in both the groups (2.63 \pm 0.62 in SA and 2.57 \pm 0.49 in GA, P value 0.647$)$. The mean duration of surgery was also comparable between two groups (89.56 \pm 29.42 minutes in SA and 86.67 \pm 31.07 minutes in GA, P value 0.694). Among the spinal anaesthesia group, 6(21.42\%) had shoulder pain, 2(7.14\%) had intraoperative nausea/vomiting, 9(32.14\%) had hypertension. None of the GA group had intraoperative complications. The proportion of subjects with a post-operative headache was $25 \%$ and $17.78 \%$ in SA and GA group respectively (P value 0.61 ). The proportion of people with postoperative nausea and vomiting was $14.2 \%$ and $37.7 \% \%$ in SA and GA groups respectively (P value 0.031 ). Conclusions: Spinalanaesthesia is a safe and effective alternative to general anaesthesia to perform laparoscopic cholecystectomy.
\end{abstract}

Keywords: Laparoscopic cholecystectomy, Spinal anaesthesia, Efficacy

\section{Introduction}

Symptomatic gallstone disease is one of the most common disease encountered in surgical practice [1]. Hence cholecystectomy is one of the most common surgical procedures performed by surgeons globally and in India[2]. Considering the rapid rise in the prevalence of various risk factors for gallstonediseases like obesity, alcoholism, the incidence of the disease and number of surgeries has been on the rise in recent years[3]. Laparoscopic cholecystectomy has gradually replaced and emerged as the gold standard for surgical

Manuscript Received: $24^{\text {th }}$ February 2018

Reviewed: $4^{\text {th }}$ March 2018

Author Corrected: $11^{\text {th }}$ March 2018

Accepted for Publication: $16^{\text {th }}$ March 2018 management of symptomatic gallstone disease. As per reports as high as $98 \%$ of the cholecystectomies are being performed by this minimal access technique in some of the nations[4]. Various advantages like relatively lesser tissue dissection, lesser disruption of tissue planes, Lesser post-operative pain, Lower incidence of Intra and postoperative complications, early return to work and the Superior cosmetic outcome makes it an obvious choice over open cholecystectomy in the majority of the cases[5,6].

Traditionally, the laparoscopic cholecystectomy has been performed under general anaesthesia [7]. But 
Original Research Article

many studies in recent times have proposed spinal anaesthesia as a better alternative to general anaesthesia in terms of achieving better postoperative analgesia. Many studies have proposed it to be a more suitable alternative in resource poor settings like India, considering lower cost and possibility of shorter hospital stay[8-10] but there are some concerns regarding spinal anaesthesia including intraoperative shoulder pain, post spinal headache, post-operative nausea, and vomiting etc $[11,12]$. Since the number of available studies on the subject is limited, clear evidence-based guidelines have not emerged in this regard. Hence there is a strong need for further studies on the subject, especially on the Indian population. In this background, the current study was conducted with the following objectives.

\section{Objectives}

1. To compare the efficacy of spinal anesthesia and general anesthesia in patients undergoing laparoscopic cholecystectomy

2. To compare the intra and postoperative complications between the two study groups

\section{Materials and methods}

Study design: Prospective observational study

Study setting: The study was conducted in the department of general surgery and department of anesthesia, Velammal medical college and Hospital, Madurai, which is a tertiary care teaching hospital.

Study period: The data collection for the study was done between April to December 2017.

Study population: The study population included patients, who were presenting to the department of general surgery OPD or emergency department subsequently posted for elective laparoscopic cholecystectomy.

\section{Inclusion criteria}

1. Adults aged more than 18 years

2. Both genders

3. ASA grade I and II

4. Clinically and radiologically confirmed cases

Exclusion criteria- Children below 18 years

All the patients were selected for the study by convenient sampling. The choice of anesthetic technique was based on the participants choice, after explaining the advantages and disadvantages of each anesthetic technique and the cost. Any patient who had a prior contraindication for any of the anesthetic technique were excluded from the study.

Ethical considerations: The study was approved by Institutional Human ethics committee. Informed written consent was obtained from all the study participants after explaining the risks and benefits of participation in the study. Confidentiality of the personal data has been maintained throughout the study.

Study procedure: After obtaining the informed written consent, all the patients had detailed preoperative evaluation and preparation for surgery as per the standard hospital protocol. All the necessary hemodynamic parameters like Blood Pressure, SpO2 and heart rate were monitored in the pre-operative room and necessary medication was administered.

The spinal anesthesiawas performed in sitting posture. Lumbar puncture was done with a 25 gauge spinal needle in the L2-L3 intervertebralspace following infiltration with a local anaesthetic. Following this intrathecal injection of $0.5 \%$ bupivacaine $(3 \mathrm{ml})$ and 25 micrograms of Fentanyl was done. After putting the patient in supine position and achieving sensory block at the T4 level, the approval was given to perform surgery. If the patient did not achieve T4 level sensory block after 5 minutes of supine position, Trendelenburg position was attempted and if at the end of $20 \mathrm{~min}$ if adequate sensory block level was not achieved, the patient was converted to GA.

All necessary haemodynamic parameters were measured intra operatively for every 5 minutes and if the mean arterial blood pressure (MAP) declined more than $20 \%$ below the pre-anesthetic level, it was labeled as intraoperative hypotension and managed with in termittent incremental iv boluses of Ephedrine $5 \mathrm{mg}$. If any patient complained ofshoulder pain during the course of surgery, was managed with $25 \mathrm{mcg}$ fentanyl iv bolus repeated at every 5 minutes, till a maximum dose of $50 \mathrm{mcg}$. GA was induced on the persistence of severe pain despite a maximum dose of fentanyl.

Following pre-oxygenation, the General anaesthesia group has received induction withPropofol $(2 \mathrm{mg} / \mathrm{kg})$, Fentanyl $(2 \mathrm{mcg} / \mathrm{kg}$, ) and Atracurium $(0.5 \mathrm{mg} / \mathrm{kg})$. All subjects were intubated after $3 \mathrm{~min}$ of ventilation and were maintained with air oxygen mixture enriched with Isoflurane (0.6-1.5\%) and controlled mechanical ventilation. The surgery was performed by keeping the patients in a supine, reverse Trendelenburg position 


\section{Original Research Article}

with the arms fully abducted minimal possible tilt to facilitate exposure of the gallbladder. Pneumoperitoneum was set at a pressure of $12 \mathrm{mmHg}$, initial insufflation of Carbon Dioxide (CO2) was done at a low flow rate $(2 \mathrm{~L} / \mathrm{min})$ and gradually increased to $5 \mathrm{~L} / \mathrm{min}$. The surgery was performed using standard four-trocar technique using a zero-degree optical scope. Gallbladder dissection was done as per standard protocol starting from Calot's triangle. Following surgical removal of the gallbladder, the subhepatic drain was placed, as per standard institutional protocol.

Statistical methods: The data were analyzed using IBM SPSS statistical software version 21. Descriptive analysis was done using mean and standard deviation for quantitative variables, frequencyand proportion for categorical variables. Both the study groups were compared with respect to various baseline variables like age, gender, BMI and presence of relevant comorbidities. The primary outcome variable was postoperative pain assessed by 10-point Visual analog scale (VAS). Considering its non-normal distribution within each group, it was compared between two groups using Mann Whitney $U$ test. The normally distributed quantitative variables were compared between two groups using unpaired t-test. Categorical variables were compared between two groups using Chi square test/ Fisher's exact test. $\mathrm{P}$ value $<0.05$ was considered statistically significant.

\section{Results}

A total of 73 subjects were included in the final analysis, with 28 subjects in spinal anesthesia group and 45 subjects in general anesthesia group.

Table-1: Comparison of baseline characteristics of the study population

\begin{tabular}{|c|c|c|c|}
\hline Parameter & $\begin{array}{c}\text { Group } 1 \text { (Spinal) } \\
(\mathbf{N}=\mathbf{2 8})\end{array}$ & $\begin{array}{c}\text { Group } 2 \text { (General) } \\
(\mathbf{N}=\mathbf{4 5})\end{array}$ & P value \\
\hline Age & $46.23 \pm 8.36$ & $48.46 \pm 10.21$ & 0.335 \\
\hline \multicolumn{4}{|c|}{ Gender } \\
\hline Female & $19(67.85 \%))$ & $31(68.8 \%)$ & \multirow{2}{*}{0.939} \\
\hline Male & $9(32.15 \%)$ & $14(31.11 \%)$ & \\
\hline BMI & $28.12 \pm 4.53$ & $27.65 \pm 5.31$ & 0.698 \\
\hline \multicolumn{4}{|c|}{ Presenting Symptom } \\
\hline Pain abdomen & $27(96.42 \%)$ & $45(100 \%)$ & * \\
\hline Vomiting & $20(71.42 \%)$ & $36(80 \%)$ & 0.399 \\
\hline Fever & $15(53.57 \%)$ & $20(44.44 \%)$ & 0.447 \\
\hline Jaundice & $13(46.42 \%)$ & $19(42.22 \%)$ & 0.724 \\
\hline \multicolumn{4}{|c|}{ Presence of co-morbidities } \\
\hline Diabetes mellitus & $11(39.28 \%)$ & $17(37.77 \%)$ & 0.901 \\
\hline Hypertension & $8(28.57 \%)$ & $12(26.66 \%)$ & 0.860 \\
\hline \multicolumn{4}{|c|}{ ASA grade } \\
\hline ASA grade I & $23(82.15 \%)$ & $36(80 \%)$ & \multirow{2}{*}{0.821} \\
\hline ASA Grade II & $5(17.85 \%)$ & $9(20 \%)$ & \\
\hline
\end{tabular}

*No statistical test was applied-due to 0 subjects in the cells.

Among the study population, the mean age of spinal anaesthesia group was $46.23 \pm 8.36$ and $48.46 \pm 10.21$ in general anaesthesia group. The difference between age and study groups was statistically not significant $(\mathrm{P}$ value 0.335$)$. In both,thestudy groups the proportion of females was almost double as that of males, but no statistically significant differences were found between two groups. No statistically significant differences were found between the two study groups, in terms of Anthropometric parameters, presenting symptoms, the presence of comorbidities and ASA grade (Table 1) 
Original Research Article

Table-2: Comparison of baseline characteristics of the study population.

\begin{tabular}{|c|c|c|c|}
\hline Parameter & Group 1 (Spinal)(N=28) & Group 2 (General)(N=45) & P value \\
\hline Ease of operating conditions & $2.63 \pm 0.62$ & $2.57 \pm 0.49$ & 0.647 \\
\hline Duration of surgery in minutes & $89.56 \pm 29.42$ & $86.67 \pm 31.07$ & 0.694 \\
\hline \multicolumn{3}{|c|}{ Other Intraoperative events } & $*$ \\
\hline Shoulder pain & $6(21.42 \%)$ & $0(0.00 \%)$ & $*$ \\
\hline Intraoperative nausea/Vomiting & $2(7.14 \%)$ & $0(0.00 \%)$ & $*$ \\
\hline Hypotension & $9(32.14 \%)$ & $0(0.00 \%)$ & $*$ \\
\hline Conversion to GA & $0(0.00 \%)$ & NA & $*$ \\
\hline
\end{tabular}

*No statistical test was applied-due to 0 subjects in the cells.

Among the mean ease of operating conditions of spinal anaesthesia group was $2.63 \pm 0.62$ and among the general anaesthesia group, $2.57 \pm 0.49$. The difference between study groups and ease of operating conditions was statistically not significant (P value 0.647).

Among the mean duration of surgery of spinal anaesthesia group, was $89.56 \pm 29.42$ and among the general anaesthesia group, 86.67 \pm 31.07 . The difference between study groups and duration of surgery was statistically not significant ( $\mathrm{P}$ value 0.694). Among the spinal anaesthesia group, 6(21.42\%) had shoulder pain, 2(7.14\%) had intraoperative nausea/vomiting, 9(32.14\%) had hypertension. (Table 2)

Table-3: Comparison of Post-operative analgesia and use of adjuvant analgesic between two study groups

\begin{tabular}{|c|c|c|c|}
\hline $\begin{array}{c}\text { Postoperative analgesia (As assessed } \\
\text { by VAS score) }\end{array}$ & $\begin{array}{l}\text { Group } 1 \text { (Spinal) } \\
\qquad(\mathbf{N}=\mathbf{3 0})\end{array}$ & $\begin{array}{c}\text { Group } 2 \text { (General) } \\
\qquad(\mathbf{N}=\mathbf{3 0})\end{array}$ & $P$ value \\
\hline Immediate post-operative period & $0(0,1)$ & $3(2,6)$ & $<0.001$ \\
\hline 30 minutes & $0(0,1)$ & $3(2,6)$ & $<0.001$ \\
\hline 60 minutes & $0(0,1)$ & $4(2,7)$ & $<0.001$ \\
\hline 90 minutes & $0(0,1)$ & $4.5(2,8)$ & $<0.001$ \\
\hline 120 minutes & $1(1,2)$ & $4.5(2,8)$ & $<0.001$ \\
\hline 4 hours & $2(1,3)$ & $4.5(2,8)$ & $<0.001$ \\
\hline 8 hours & $3.5(2,5)$ & $4(2,7)$ & 0.326 \\
\hline 10 hours & $4(2,6)$ & $3.5(2,6)$ & 0.412 \\
\hline 12hours & $5(2,8)$ & $4.5(2,8)$ & 0.512 \\
\hline 24 hours & $4.5(2,8)$ & $4.5(2,8)$ & 1 \\
\hline
\end{tabular}

Among the spinal anaesthesia median VAS was $0(\mathrm{IQR} 0$ to1) at immediate post-operative period, $0(\mathrm{IQR} 0$ to 1$)$ at 30 minutes, 0 (IQR 0 to 1) 0f 60 minutes, 0 (IQR0 to 1) of 90 minutes, 1 (IQR1 to 2) 0f 120 minutes, 2(IQR 1 to 30 ) of 4 hours, 3.5 (IQR 2 TO 5) OF 8 hours, 4 (IQR 2 to 6) 0f 10 hours, 5(IQR 2 to 8) of 12 hours, 4.5(IQR 2 to 8 ) of 24 hours. Among the general anaesthesia median was $3(\mathrm{IQR} 2$ to 6 ) of immediate post-operative period, 3(IQR 2 to 6 ) of 30 minutes, 4(IQR 2 to 7) 0f 60 minutes, 4.5(IQR 2 to 8 ) of 90 minutes, 4.5(IQR 2 to 8 ) of 120 minutes, 4.5(IQR 2 to 8 ) of 4 hours, 4 (IQR 2 to 7) of 8 hours, 3.5(IQR 2 to 6) of 10 hours, 4.5(IQR 2 to 8 ) of 12 hours, $4.5(2$ to 8 ) 0 f 24 hours preoperative analgesia.

The difference across study groups and immediate post-operative period,30,60,90,120 minutes, 4 hours were statistically significant $(\mathrm{P}$ value $<0.001)$, The difference across study groups and 8,10,12,24 hours' post-operative analgesia was statistically not significant ( $\mathrm{P}$ value $>0.05$ ) (Table 3 ). 
Original Research Article

Table-4: Incidence of various post-operative complications between two study groups.

\begin{tabular}{|c|c|c|c|}
\hline Post operative Complications & Group 1 (Spinal)(N=28) & Group 2 (General)(N=45) & P value \\
\hline Post-operative headache & $7(25 \%)$ & $9(17.78 \%)$ & 0.61 \\
\hline Post-operative nausea and vomiting & $4(14.2 \%)$ & $17(37.78 \%)$ & 0.031 \\
\hline Post-operative wound sepsis & $0(0.00 \%)$ & $0(0.00 \%)$ & ND \\
\hline
\end{tabular}

*ND+ not done as the data did not satisfy assumptions required to carry out chi square test/Fisher's exact test

Among the spinal anaesthesia group, 7 (25\%) people had a post-operative headache, which was 9 (17.78\%) in general anesthesia group and the difference was statistically not significant ( $\mathrm{P}$ value 0.61 ). The proportion of people with postoperative nausea and vomiting was $14.2 \%$ and $37.7 \% \%$ in SA and GA groups respectively, with a statistically significant difference (0.031). None of the subjects in either of treatment groups had postoperative wound sepsis (Table 4).

\section{Discussion}

Laparoscopic Cholecystectomy has emerged as the safe and effective choice over open cholecystectomy in recent years. Even in developing countries,amajor portion of cholecystectomies is being performed laparoscopically [5].

In the current study, the operating time and ease of operating conditions and duration of hospital stay etc were comparable between both spinal and general anaesthesia groups. These findings were in line with the majority of the published literature on the subject. None of the subjects in spinal anaesthesia group required conversion to general anaesthesia. Tzovaras, G., et al.[13] In one of the first pilot study on the feasibility of spinal anaesthesia for Laparoscopic cholecystectomy have reported $100 \%$ successful execution.Yuksek, Y. N., et al.[12], have reported a conversion rate of $11.53 \%$ of cases to general anaesthesia due to severe right shoulder pain. Developed during spinal anesthesia. Gautam, B et al[8] have reported the failure very minimal operative difficulty. In a study by Sinha, R., et $\mathrm{al}[10]$ among laparoscopic cholecystectomy cases done under spinal anaesthesia, $0.52 \%$ patients required a conversion to GA. There were no differences between two study groups in operating time and other operative parameters.

In the current study, The spinal anaesthesia had resulted in better postoperative analgesia, especially in the first 4-hour post-operative period. The overall analgesic requirement was also lesser in this group, as compared to general anaesthesia. Tzovaras, G., et al.[13] have reported a postoperative median pain scores ranging from 1 to 1.5 , with no major complications with spinal anesthesia.

Similar to current study findings, Tzovaras, G., et al. [14] in another study have reported that significantly lower pain until 4 hours. But in contrast to the current study, the superior painrelief with spinal anaesthesia had continued till 24 hours. In a study by Sinha, R., et al.[10] the overall post-operative injectable analgesic requirement was $34.36 \%$ and $91.45 \%$ in SA and GA groups respectively, indicating superior pain relief with SA. Bessa, S. S., et al.[15] have also reported similar findings to the current study, where for the first 2 to 4 hours, the mean pain scores were significantly lesser with spinal anaesthesia, as compared to general anaesthesia. The analgesic requirement in the first postoperative day was also significantly lower in SA group. Imbelloni, L. E., et al. [16] have reported significantly lower pain with spinal anaesthesia in the first 6 hours following surgery.

In the current study, among the spinal anaesthesia group, $6(21.42 \%)$ had shoulder pain, $2(7.14 \%)$ had intraoperative nausea/vomiting and $9(32.14 \%)$ had hypertension. None of the subjects in general anaesthesia group had the above-mentioned complications. In a study by Yuksek, Y. N., et al.[12], out of 26 patients attempted for laparoscopic cholecystectomy under SA, 3 patients needed conversion to general anesthesia due to severe right shoulder pain. No major cardiopulmonary problems were reported by this study except transient hypotension Intra operatively. But almost $50 \%$ of the subjects had reported severe right shoulder pain, needing treatment by fentanyl, local washing of the right diaphragm with $2 \%$ lidocaine solution. As per a study by Gautam, B et al [8]the problems reported in the spinal anesthesia group were shoulder pain intraoperatively requiring treatment with by Fentanyl and anxietytreated by midazolam.Inastudy by Sinha, R., et al[10] among laparoscopic cholecystectomy cases done under spinal anaesthesia, the most common issues occurred during the intraoperative period were hypotension requiring 


\section{Original Research Article}

treatment in $20.05 \%$ of patients and, neck and/or shoulder pain in $12.29 \%$ of patients. Which was similar to current study findings. In a study by Kumar, A et al[9] commonest complaint reported was a pain in right shoulder and anxiety at the beginning of operation/ pneumoperitoneum. In the current study Among the spinal anaesthesia group, $7(25 \%)$ people had a postoperative headache, which was $9(17.78 \%)$ in general anesthesia group and the difference was statistically not significant ( $\mathrm{P}$ value 0.61 ). The proportion of people with post-operative nausea and vomiting was $14.2 \%$ and $37.7 \%$ in SA and GA groups respectively, with a statistically significant difference (0.031). None of the subjects in either of treatment groups had postoperative wound sepsis. Tzovaras, G., et al[14]in contrast to current study have reported comparable post-operative complication rate between spinal and general anesthesia. In a study by Sinha, R., et al[10] postoperative nausea and vomiting were present in $2.29 \%$ in SA group against $30.30 \%$ in GA. A postural headache was reported in $5.9 \%$ of patients following SA.

A randomized controlled trial by Bessa, S. S., et al[17] have reported higher proportion $(8.8 \% \mathrm{Vs} 0 \%)$ of subjects in GA group requiring anovernight postoperative stay, as they had nausea and vomiting, inadequate pain control and unexplained hypotension.

Imbelloni, L. E., et al[16] have reported significantly lower pain with spinal anaesthesia in the first 6 hours following surgery. They have also specified the cost of spinal anaesthesia to be considerably lower as compared to GA and proposed it to be a more suitable option in resource-limitedsettings.

\section{Conclusions}

1. The spinal anaesthesia had resulted in better postoperative analgesia, especially in the first 4-hour post-operative period.The overall analgesic requirement was also lesser in this group, as compared to general anaesthesia.

2. The incidence of some of the intraoperative complications like shoulder pain and intraoperative hypotension, nausea, and vomiting were slightly higher in spinal anaesthesia group. A post-operative headache also was slightly higher in spinal anaesthesia group.

3. The incidence of postoperative nausea and vomiting was higher in general anaesthesia grou.

4. The operating time, operating conditions and postoperative stay were comparable between two groups.
Limitations: Since the study participant selection was not randomly done, the role of selection bias and the effect of potential unknown confounding factors and the magnitude and direction of their effect could not be estimated

Recommendations: There is a need for large-scale randomized controlled trials on the subject to generate more evidence on the subject to guide informed clinical decision making. Till such time the choice of anaesthesia should be made by carefully weighing the risks, benefits, cost of the procedure and patient preferences into consideration.

What this study adds to existing knowledge: The study further strengthens the evidence existing regarding the comparative efficacy and safety of spinal anaesthesia in patients undergoing laparotomy.

The study findings prove that the spinal anaesthesia can be used safely for laparoscopic cholecystectomy and achieve comparative efficacy, without any undue adverse effects. The study findings assume greater importance due to scarcity of studies on the subject in Indian population.

\section{Author Contribution}

Author 1: The first author has conceptualized the study, participated in developing appropriate study protocol, involved in all stages of the study from the design, data collection, data analysis, preparation and review of the drafts to submission of the manuscript for publication.

Author 2: The second author had provided a supportive role data collection, data entry. He had contributed to the preparation of the draft manuscript, editing of the drafts and also was involved in doing necessary modifications to the drafts as per reviewer'sinputs.

Conflict of interest: None declared. Funding: Nil, Permission from IRB: Yes

\section{References}

1. Demehri FR, Alam HB. Evidence-Based Management of Common Gallstone-Related Emergencies. J Intensive Care Med. 2016;31(1):3-13. doi: 10. 1177/ 0885066614554192 .

2. Stinton LM, Shaffer EA. Epidemiology of gallbladder disease: cholelithiasis and cancer. Gut Liver. 2012 Apr; 6(2): 172-87. doi: 10.5009/gnl. 2012. 6.2. 172. Epub 2012 Apr 17. 


\section{Original Research Article}

3. Shaffer EA. Gallstone disease: Epidemiology of gallbladder stone disease. Best Pract Res Clin Gastroenterol. 2006;20(6):981-96. doi: 10.1016/j. bpg. 2006. 05.004.

4. Tazuma S, Unno M, Igarashi Y, Inui K, Uchiyama K, Kai M, et al. Evidence-based clinical practice guidelines for cholelithiasis 2016. J Gastroenterol. 2017;52(3):276300. doi: 10.1007/s00535-016-1289-7

5. Bal S, Reddy LG, Parshad R, Guleria R, Kashyap L. Feasibility and safety of day care laparoscopic cholecystectomy in a developing country. Postgrad Med J. 2003;79(931):284-8. doi: 10.1136/pmj.79.931.284

6. Sedaghat N, Cao AM, Eslick GD, Cox MR. Laparoscopic versus open cholecystectomy in pregnancy: a systematic review and meta-analysis. Surg Endosc. 2017; 31(2):673-9. doi: 10.1007/s00464-0165019-2

7. Watanabe Y, Bilgic E, Lebedeva E, McKendy KM, Feldman LS, Fried GM, et al. A systematic review of performance assessment tools for laparoscopic cholecystectomy. SurgEndosc. 2016; 30(3):832-44. doi: 10. 1007/s00464-015-4285-8

8. Gautam B. Spinal anaesthesia for laparoscopic cholecystectomy: a feasibility and safety study. Kathmandu Univ Med J (KUMJ). 2009; 7 (28): 360-8. doi: 10.3126/kumj.v7i4.2755

9. Kumar A. Laparoscopic cholecystectomy under spinal anaesthesia: a prospective study. Nepal Med Coll J. 2014; 16(2-4):139-43. doi: 10. 9790 /0853-150905 117120

10. Sinha R, Gurwara AK, GuptaSC. Laparoscopic cholecystectomy under spinal anesthesia:

Astudy of 3492 patients. J Laparoendosc Adv Surg Tech A. 2009 Jun; 19(3): 323-7. doi: 10.1089/ lap. 2008.0393 .
11. Yousef GT, Lasheen AE. General anesthesia versus segmental thoracic or conventional lumbar spinal anesthesia for patients undergoing laparoscopic cholecystectomy. Anesth Essays Res. 2012;6(2):167-73. doi: 10.4103/0259-1162.108302.

12. Yuksek YN, Akat AZ, Gozalan U, Daglar G, Pala Y, Canturk M, et al. Laparoscopic cholecystectomy under spinal anesthesia. Am J Surg. 2008;195(4):533-6. doi: 10.1016/j.amjsurg.2007.05.043

13. Tzovaras G, Fafoulakis F, Pratsas K, Georgopoulou S, Stamatiou G, Hatzitheofilou C. Spinal vs general anesthesia for laparoscopic cholecystectomy: interim analysis of a controlled randomized trial. Arch Surg. 2008 May; 143 (5): 497-501. doi: 10.1001/archsurg. 143.5.497.

14. Tzovaras G, Fafoulakis F, Pratsas K, Georgopoulou S, Stamatiou G, Hatzitheofilou C. Spinal vs general anesthesia for laparoscopic cholecystectomy: interim analysis of a controlled randomized trial. Arch Surg. 2008 May; 143(5): 497-501. doi: 10.1001/ archsurg. 143.5.497.

15. Bessa SS, El-Sayes IA, El-Saiedi MK, Abdel-Baki NA, Abdel-Maksoud MM. Laparoscopic cholecystectomy under spinal versus general anesthesia: a prospective, randomized study. J LaparoendoscAdvSurg Tech A. 2010;20(6):515-20. doi: 10.1089/lap.2010.0041

16. Imbelloni LE, Fornasari M, Fialho JC, Sant'Anna R, Cordeiro JA. General anesthesia versusspinalanesthesia for laparoscopic cholecystectomy. Rev Bras Anestesiol. 2010 May-Jun;60(3):217-27. doi: 10.1016/S0034-7094 (10) $70030-1$.

17. Bessa SS, Katri KM, Abdel-Salam WN, El-Kayal el SA, Tawfik TA. Spinal versus general anesthesia for day-case laparoscopic cholecystectomy: a prospective randomized study. J Laparoendosc Adv Surg Tech A. 2012; 22(6):550-5. doi: 10.1089/lap.2012.0110

\section{How to cite this article?}

Subbiah V, Palaniappan S.K. A prospective observational study to compare the efficacy and safety of spinal Vs general anesthesia for laparoscopic cholecystectomy. Surgical Update: Int J surg Orthopedics.2018;4(1):45-51.doi:10. 17511/ijoso.2018.i01.08. 